International Mathematical Forum, Vol. 8, 2013, no. 19, 945 - 948

HIKARI Ltd, www.m-hikari.com

\title{
Asymptotic Formulae for the Square Root of the $n$-th Perfect Power
}

\author{
Rafael Jakimczuk \\ División Matemática, Universidad Nacional de Luján \\ Buenos Aires, Argentina \\ jakimczu@mail.unlu.edu.ar
}

Copyright (C) 2013 Rafael Jakimczuk. This is an open access article distributed under the Creative Commons Attribution License, which permits unrestricted use, distribution, and reproduction in any medium, provided the original work is properly cited.

\begin{abstract}
In this note we obtain asymptotic formulae for the square root of the $n$-th perfect power.
\end{abstract}

Mathematics Subject Classification: 11A99, 11B99

Keywords: Asymptotic formulae, $n$-th perfect power, square root

\section{Introduction and Lemmas}

A natural number of the form $m^{n}$ where $m$ is a positive integer and $n \geq 2$ is called a perfect power. The first few terms of the integer sequence of perfect powers are

$$
1,4,8,9,16,25,27,32,36,49,64,81,100,121,125,128 \ldots
$$

and they are sequence A001597 in Sloane's Encyclopedia. In this note $P_{n}$ denotes the $\mathrm{n}$-th perfect power.

A quadratfrei number is a number without square factors, a product of different primes. The first few terms of the integer sequence of quadratfrei numbers are

$$
1,2,3,5,6,7,10,11,13,14,15,17,19,21,22,23,26,29,30, \ldots
$$

On the other hand, the Möbius function $\mu(n)$ is defined as follows: $\mu(1)=1$, if $n$ is the product of $r$ different primes, then $\mu(n)=(-1)^{r}$, if the square of a 
prime divides $n$, then $\mu(n)=0$. In this note $q$ denotes a quadratfrei number. On the other hand (as usual) $p_{n}$ denotes the $n$-th prime number.

We shall need the following theorem.

Theorem 1.1 Let $p_{h}$ be the $h$-th prime with $h \geq 3$, where $h$ is an arbitrary but fixed positive integer. We have the following asymptotic formula

$$
P_{n}=n^{2}+\frac{13}{3} n^{8 / 6}+\frac{32}{15} n^{32 / 30}+\left(\sum_{\substack{2 \leq q \leq p_{h} \\ q \neq 2,6,30}} 2 \mu(q) n^{1+\frac{2}{q}}\right)+o\left(n^{1+\frac{2}{p_{h}}}\right)
$$

Note that $2=1+\frac{2}{2}, \frac{8}{6}=1+\frac{2}{6}$ and $\frac{32}{30}=1+\frac{2}{30}$.

Proof. See [1].

If $h=3$ then Theorem 1.1 becomes

$$
P_{n}=n^{2}-2 n^{\frac{5}{3}}-2 n^{\frac{7}{5}}+o\left(n^{\frac{7}{5}}\right) .
$$

If $h=4$ then Theorem 1.1 becomes

$$
P_{n}=n^{2}-2 n^{\frac{5}{3}}-2 n^{\frac{7}{5}}+\frac{13}{3} n^{\frac{4}{3}}-2 n^{\frac{9}{7}}+o\left(n^{\frac{9}{7}}\right) .
$$

If $h=5$ then Theorem 1.1 becomes

$$
P_{n}=n^{2}-2 n^{\frac{5}{3}}-2 n^{\frac{7}{5}}+\frac{13}{3} n^{\frac{4}{3}}-2 n^{\frac{9}{7}}+2 n^{\frac{6}{5}}-2 n^{\frac{13}{11}}+o\left(n^{\frac{13}{11}}\right) .
$$

The following lemma is a immediate consequence of the binomial theorem.

Lemma 1.2 We have the following formulae

$$
\begin{gathered}
(1+x)^{1 / 2}=1+\frac{1}{2} x+O\left(x^{2}\right) \quad(x \rightarrow 0) \\
(1+x)^{1 / 2}=1+\frac{1}{2} x+\frac{\frac{1}{2}\left(\frac{1}{2}-1\right)}{2} x^{2}+O\left(x^{3}\right)=1+\frac{1}{2} x-\frac{1}{8} x^{2}+O\left(x^{3}\right) \quad(x \rightarrow 0)
\end{gathered}
$$

In this note we obtain asymptotic formulae for $\sqrt{P_{n}}$.

\section{Main Results}

Theorem 2.1 Let $p_{h}$ be the $h$-th prime with $h \geq 3$, where $h$ is an arbitrary but fixed positive integer. We have the following asymptotic formula

$$
\sqrt{P_{n}}=n+\frac{5}{3} n^{2 / 6}+\frac{1}{15} n^{2 / 30}+\sum_{\substack{2 \leq q \leq p_{h} \\ q \neq 2,6,30}} \mu(q) n^{2 / q}+o\left(n^{2 / p_{h}}\right)
$$


Proof. First, we shall prove the theorem if $h=3$, that is $p_{h}=5$. We have (see (2))

$P_{n}=n^{2}-2 n^{1+\frac{2}{3}}-2 n^{1+\frac{2}{5}}+o\left(n^{1+\frac{2}{5}}\right)=n^{2}\left(1-2 n^{-1+\frac{2}{3}}-2 n^{-1+\frac{2}{5}}+o\left(n^{-1+\frac{2}{5}}\right)\right)$

Therefore

$$
\sqrt{P_{n}}=n\left(1-2 n^{-1+\frac{2}{3}}-2 n^{-1+\frac{2}{5}}+o\left(n^{-1+\frac{2}{5}}\right)\right)^{1 / 2}=n(1+x)^{1 / 2}
$$

where

$$
x=-2 n^{-1+\frac{2}{3}}-2 n^{-1+\frac{2}{5}}+o\left(n^{-1+\frac{2}{5}}\right) \sim-2 n^{-1 / 3}
$$

Equations (7), (8) and (4) give

$$
\begin{aligned}
\sqrt{P_{n}} & =n\left(1+\frac{1}{2}\left(-2 n^{-1+\frac{2}{3}}-2 n^{-1+\frac{2}{5}}+o\left(n^{-1+\frac{2}{5}}\right)\right)+O\left(n^{-2 / 3}\right)\right) \\
& =n-n^{2 / 3}-n^{2 / 5}+o\left(n^{2 / 5}\right)+O\left(n^{2 / 6}\right)=n-n^{2 / 3}-n^{2 / 5}+o\left(n^{2 / 5}\right)
\end{aligned}
$$

That is, equation (6) if $h=3$.

If $h \geq 4$, that is $p_{h} \geq 7$, equation (1) can be written in the form (see (3))

$$
P_{n}=n^{2}-2 n^{1+\frac{2}{3}}-2 n^{1+\frac{2}{5}}+\frac{13}{3} n^{1+\frac{2}{6}}+\frac{32}{15} n^{1+\frac{2}{30}}+\sum_{\substack{7 \leq q \leq p_{h} \\ q \neq 30}} 2 \mu(q) n^{1+\frac{2}{q}}+o\left(n^{1+\frac{2}{p_{h}}}\right)
$$

Note that if $7 \leq p_{h} \leq 29$ equation (9) becomes

$$
P_{n}=n^{2}-2 n^{1+\frac{2}{3}}-2 n^{1+\frac{2}{5}}+\frac{13}{3} n^{1+\frac{2}{6}}+\sum_{\substack{7 \leq q \leq p_{h} \\ q \neq 30}} 2 \mu(q) n^{1+\frac{2}{q}}+o\left(n^{1+\frac{2}{p_{h}}}\right)
$$

Since $n^{1+\frac{2}{30}}=o\left(n^{1+\frac{2}{p_{h}}}\right)$.

Equation (9) gives

$$
P_{n}=n^{2}(1+x)
$$

where

$$
\begin{aligned}
x & =-2 n^{-1+\frac{2}{3}}-2 n^{-1+\frac{2}{5}}+\frac{13}{3} n^{-1+\frac{2}{6}}+\frac{32}{15} n^{-1+\frac{2}{30}}+\sum_{\substack{7 \leq q \leq p_{h} \\
q \neq 30}} 2 \mu(q) n^{-1+\frac{2}{q}} \\
& +o\left(n^{-1+\frac{2}{p_{h}}}\right) \sim-2 n^{-1 / 3}
\end{aligned}
$$

Equations (10) and (5) give

$$
\begin{aligned}
\sqrt{P_{n}} & =n(1+x)^{1 / 2}=n\left(1+\frac{1}{2} x-\frac{1}{8} x^{2}+O\left(x^{3}\right)\right) \\
& =n+\frac{1}{2} n x-\frac{1}{8} n x^{2}+n O\left(x^{3}\right)
\end{aligned}
$$


Equation (11) gives

$$
\begin{aligned}
& n O\left(x^{3}\right)=n O\left(n^{-1}\right)=O(1)=o\left(n^{2 / p_{h}}\right) \\
& \frac{1}{2} n x=-n^{2 / 3}-n^{2 / 5}+\frac{13}{6} n^{2 / 6}+\frac{16}{15} n^{2 / 30}+\sum_{\substack{7 \leq q \leq p_{h} \\
q \neq 30}} \mu(q) n^{2 / q}+o\left(n^{2 / p_{h}}\right) \\
& -\frac{1}{8} n x^{2} \\
& =-\frac{1}{8} n\left(-2 n^{-1+\frac{2}{3}}-2 n^{-1+\frac{2}{5}}+\frac{13}{3} n^{-1+\frac{2}{6}}+\frac{32}{15} n^{-1+\frac{2}{30}}+\sum_{\substack{7 \leq q \leq p_{h} \\
q \neq 30}} 2 \mu(q) n^{-1+\frac{2}{q}}\right)^{2} \\
& +o(1)=-\frac{1}{8} n\left(-2 n^{-1+\frac{2}{3}}\right)^{2}-\frac{1}{8} n 2\left(-2 n^{-1+\frac{2}{3}}\right)\left(-2 n^{-1+\frac{2}{5}}\right)+O(1)+o(1) \\
& =-\frac{1}{2} n^{2 / 6}-n^{2 / 30}+o\left(n^{2 / p_{h}}\right)
\end{aligned}
$$

Substituting (13), (14) and (15) into (12) we find that

$$
\begin{aligned}
\sqrt{P_{n}} & =n-n^{2 / 3}-n^{2 / 5}+\frac{5}{3} n^{2 / 6}+\frac{1}{15} n^{2 / 30}+\sum_{\substack{7 \leq q \leq p_{h} \\
q \neq 30}} \mu(q) n^{2 / q}+o\left(n^{2 / p_{h}}\right) \\
& =n+\frac{5}{3} n^{2 / 6}+\frac{1}{15} n^{2 / 30}+\sum_{\substack{2 \leq q \leq p_{h} \\
q \neq 2,6,30}} \mu(q) n^{2 / q}+o\left(n^{2 / p_{h}}\right)
\end{aligned}
$$

That is, equation (6). The theorem is proved.

ACKNOWLEDGEMENTS. The author is very grateful to Universidad Nacional de Luján.

\section{References}

[1] R. Jakimczuk, Asymptotic formulae for the $n$-th perfect power, J. Integer Seq. 15 (2012), Article 12.5.5.

Received: March 29, 2013 